\title{
INFLUENCIA DE LA SILVICULTURA EN LA INCIDENCIA DE Dendroctonus frontalis, CAMPAMENTO, OLANCHO, MEDIANTE TECNOLOGÍAS DE INFORMACIÓN GEOGRÁFICA, 2000-2016.
}

\author{
Javier-Enrique Meza-Antúnez ${ }^{1, *}$ \\ ${ }^{1}$ Departamento de Ciencia y Tecnologías de la Información Geográfica, UNAH \\ Recibido: 12/junio/2019 \\ Aceptado: 04/noviembre/2019 \\ DOI: https://doi.org/10.5377/ce.v12i1.9639
}

\begin{abstract}
RESUMEN
La presente la investigación se enmarco en desarrollar una respuesta científica a la problemática del gorgojo descortezador del pino, realizando una comparación entre los bosques de que han sido tratados bajo silvicultura versus los bosques que no han recibido ningún tratamiento silvicultural en el municipio de Campamento, Olancho, para determinar la influencia de la silvicultura en estos brotes de plaga de Dendroctonus frontalis. El objetivo general consistió en analizar las variaciones en la incidencia de la silvicultura el impacto de plaga de gorgojo descortezador del pino Dendroctonus frontalis, determinado mediante Tecnologías de la Información Geográfica en el municipio de Campamento, Olancho, en el periodo 2000-2016. Este análisis se realizó utilizando las herramientas tecnológicas como los Sistemas de Información Geográfica, para determinar si hay diferencias en los daños causados por el gorgojo descortezador del pino. Se utilizó herramientas de análisis espacial disponibles en los softwares especializados, se realizó mediante las metodologías y técnicas del análisis espacial de los datos geográficos. Se pudo concluir que existe una variación porcentual en la incidencia de la silvicultura de $19.86 \%$ en el área afectada por plaga bosque de pino y bosque mixto sin manejo forestal en el área de estudio equivalente a 466.52 hectáreas. Además, existe una variación porcentual en la incidencia de la silvicultura de $14.54 \%$ en el área afectada por plaga bosque de pino y bosque mixto con manejo forestal en el área de estudio equivalente a 1959.65 hectáreas.
\end{abstract}

Palabras clave: Sistemas de Información Geográfica, Análisis espacial, autocorrelación espacial, asociación espacial, silvicultura.

\begin{abstract}
The present research aims to develop a scientific response to the problem of the pine bark beetle, making a comparison between the forests that have been treated under forestry versus the forests that have not received any treatment in the municipality of Campamento, Olancho, to determine the influence of forestry on these plague outbreaks of Dendroctonus frontalis. The general objective was to analyze the variations in the incidence of silviculture the impact of the pest of bark beetle Dendroctonus frontalis pine, determined by Geographic Information Technologies in the municipality of Campamento, Olancho, in the period 2000-2016. This analysis will be done using technological tools such as Geographic Information Systems, to determine if there are differences in the damage caused by the pine bark beetle.
\end{abstract}

*javier.meza@unah.edu.hn 
To achieve these objectives, spatial analysis tools available in specialized software will be used to perform a spatial analysis of the different geographic data. The identification and analysis of the influence of silviculture on the incidence of pine bark beetle advance will be made through the methodologies and spatial analysis techniques that can be carried out using GIS. It was concluded that there is a percentage variation in the incidence of forestry of $19.86 \%$ in the area affected by pine forest plague and mixed forest without forest management in the study area equivalent to 466.52 hectares. In addition, there is a percentage variation in the incidence of forestry of $14.54 \%$ in the area affected by pine forest plague and mixed forest with forest management in the study area equivalent to 1959.65 hectares.

Keywords: Geographic information systems, apatial analysis, spatial autocorrelation, apatial association, forestry.

\section{Introducción}

Cabe decir que, por ser un evento reciente en el país, no existe un estudio actualizado en el que se determine la influencia de la silvicultura en la incidencia de plagas (Dendroctonus frontalis) en los bosques de pino, por lo que esto representa una opción de contar con un análisis que nos muestre esta problemática desde otra perspectiva, que permita encontrar nuevas alternativas de intervención para mitigar los impactos que provoca este fenómeno. El $50 \%$ del territorio hondureño está cubierto de bosques (5.4 millones de ha), del cual un $36.7 \%$ (1.94 millones de ha) corresponden a bosques de pino y un $5 \%$ (0.3 millones) a bosques que combinan pino con otras especies (ICF, 2016).

Datos del BID (2016) indican que desde el año 2012 el bosque de pino que fue afectado por la plaga del gorgojo descortezador, que hasta diciembre del 2016 había destruido aproximadamente 508,000 Ha de bosque ( $25 \%$ del área total de pino), lo que equivale a la deforestación que habría ocurrido en 110 años. Es importante resaltar que esta área no perdió por completo, esta deberá ser objeto de evaluación de regeneración natural, para terminar el grado de restauración que presente.

El área de cobertura de pino se ha visto severamente afectada por la plaga del gorgojo descortezador del pino. Así mismo, así mismo existe debilidad en la gestión para promover practicas silviculturales apropiadas que fomenten el buen manejo forestal, falta de incentivos forestales, la tala ilegal y los incendios forestales han influenciado de gran manera la perdida de cobertura del bosque de pino.

A consecuencia de los efectos del cambio climático, Honduras está atravesando una de las peores situaciones por las plagas forestales sobre los bosques de pino, en especial la del gorgojo descortezador del pino (Dendroctonus spp) que, sumándose a otras amenazas existentes como los incendios forestales y la tala ilegal, ponen en precario el disfrute de los derechos humanos y, por ello, condiciones básicas de vida digna de las y los habitantes (CONADEH, 2016).

Sobre la base de las consideraciones anteriores este estudio permitirá determinar la necesidad de crear incentivos al buen manejo forestal en las diferentes regiones forestales ubicadas a lo largo del país. Por otra parte, la investigación generará información geográfica en el tema de plagas y enfermedades forestales para el país, lo que es primordial para el buen manejo de los recursos forestales y a la vez se utilizará como un insumo para las instituciones que dirigen el Sector Forestal. A la vez será fundamental realizar acciones de restauración forestal en estas áreas afectadas para su recuperación. Por otro lado, esta investigación también ayudará a los propietarios privados de bosques de pino y servirá para la generación de nuevos proyectos de incentivos y políticas forestales nacionales para el fomento del buen manejo forestal de los bosques. 
El manejo de las plagas y la prevención de su dispersión son aspectos fundamentales para ayudar a asegurar el mantenimiento de la sanidad de los bosques y alcanzar los objetivos de la silvicultura sostenible (FAO, 2012).

Así mismo es importante entender que la silvicultura preventiva tiene el propósito de modificar la estructura del combustible disponible y así satisfacer los objetivos de protección contra incendios forestales, asociando esta protección al mejoramiento de la producción y la calidad del medio ambiente.

\section{Metodología}

\section{Área de estudio.}

El área de estudio fue el municipio de Campamento, en el departamento de Olancho, el cual tiene una extensión territorial de $379.58 \mathrm{~km}^{2}$ y está ubicado al Suroeste del departamento, limita al Norte: con los municipios de Concordia y Juticalpa, Al Sur: con el Municipio de Teupasenti en el departamento de El Paraíso, al Este con el municipio de Juticalpa y al Oeste con el municipio de Guaimaca, departamento de Francisco Morazán (ver figura 1). Campamento cuenta con 11 aldeas y 117 caseríos. Las principales actividades económicas de este municipio son: agricultura, silvicultura, caza y pesca. 


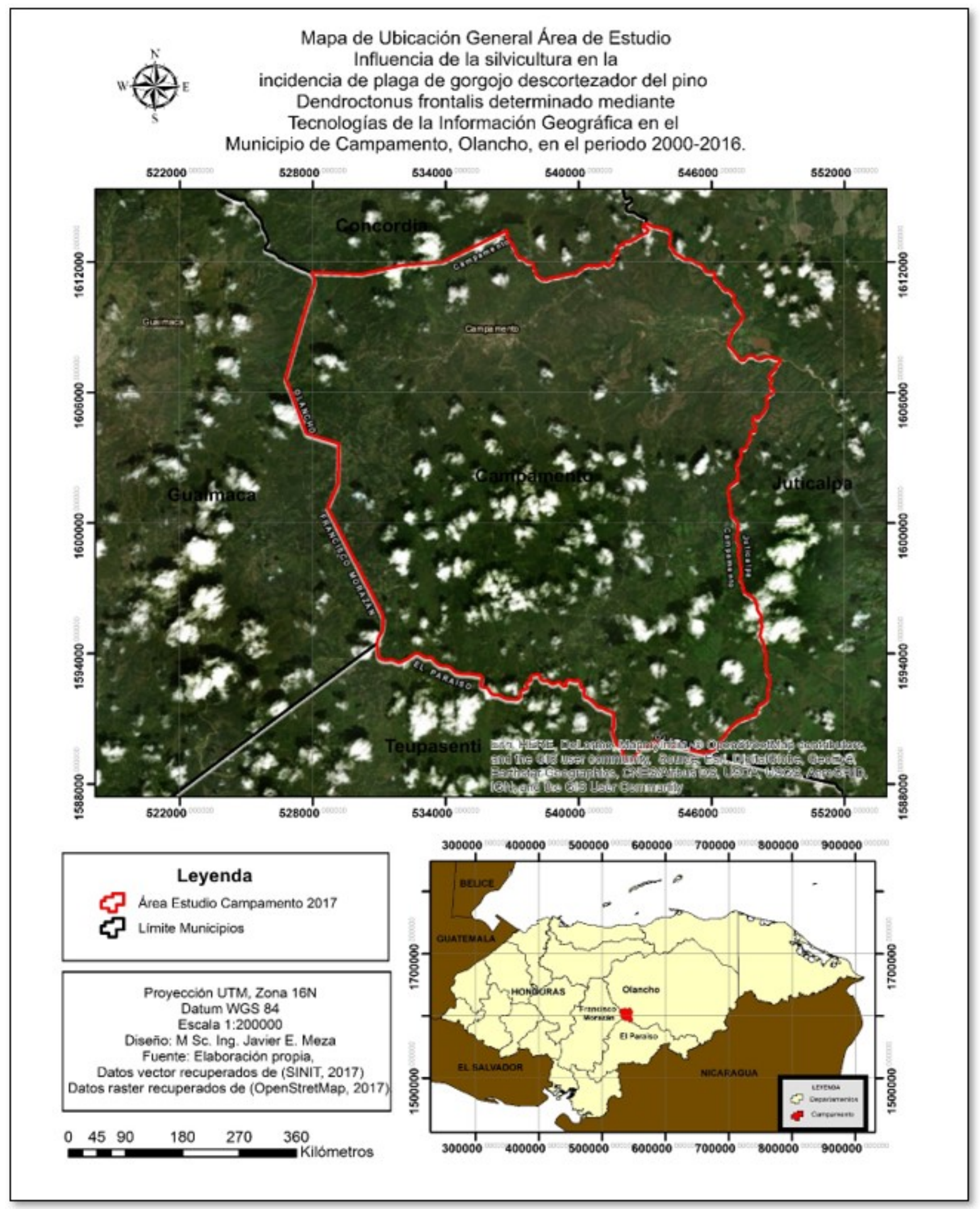

Figura 1: Mapa de ubicación área de estudio. 
Para el análisis e interpretación de los datos la investigación se realizó 4 etapas.

Etapa I: selección de la información

Esta etapa consistió en la selección de la geodatabase, diseñada en el Sistema de Información Geográfica con la base de los datos vectoriales de planes de manejo y de las áreas afectadas por la plaga en el municipio de Campamento, departamento de Olancho. En esta investigación se utilizó el formato shapefile el cual es el formato de datos vectorial más popular y extendido en el trabajo con un SIG. Se trata de un formato vectorial que guarda la localización de elementos geográficos y atributos a ellos asociados, pero no es capaz de almacenar relaciones topológicas (Alonso, 2015). La información referente utilizada en esta investigación es la siguiente:

- Cobertura/uso de la tierra: se utilizó un producto geoespacial que tuviera cubrimiento completo del contexto espacial de Honduras y específicamente el municipio de Campamento, Olancho. La fuente de datos usada procede del Mapa Forestal y de cobertura de la tierra de Honduras elaborado en 2014 por el ICF con apoyo del programa EDDS-GIZ-CAD. Imágenes de alta resolución espacial fueron utilizados para tener unidades de mapeo mínimas de 0.5 hectáreas (Hernandez, 2016).

- Capa de información geográfica de planes de manejo forestal vigentes a nivel nacional y específicamente en Olancho (ICF, 2015).

- Capa de información geográfica del área afectada por la Plaga del Gorgojo descortezador del pino Dendroctonus frontalis (ICF, 2017).

- Capa de municipios de Honduras (SINIT, 2017). Ver tabla 1: especificaciones de geodatabase

Tabla 1: Especificaciones GEODATABASE

\begin{tabular}{ll}
\hline \multicolumn{2}{c}{ Especificaciones } \\
\hline Projected Coordinate System & WGS_1984_UTM_Zone_16N \\
Projection & Transverse_Mercator \\
Geographic Coordinate System & GCS_WGS_1984 \\
Datum & D_WGS_1984 \\
Prime Meridian & Greenwich \\
Angular Unit & Degree \\
\hline
\end{tabular}

Etapa II: recopilación de la información

Se realizó una compilación de los planes de manejo de la zona de la Región Forestal ICF Olancho en donde se han realizado diferentes tratamientos silvícolas en el municipio de Campamento durante el periodo comprendido en los años 2000 a 2016 (ver figura 2). 


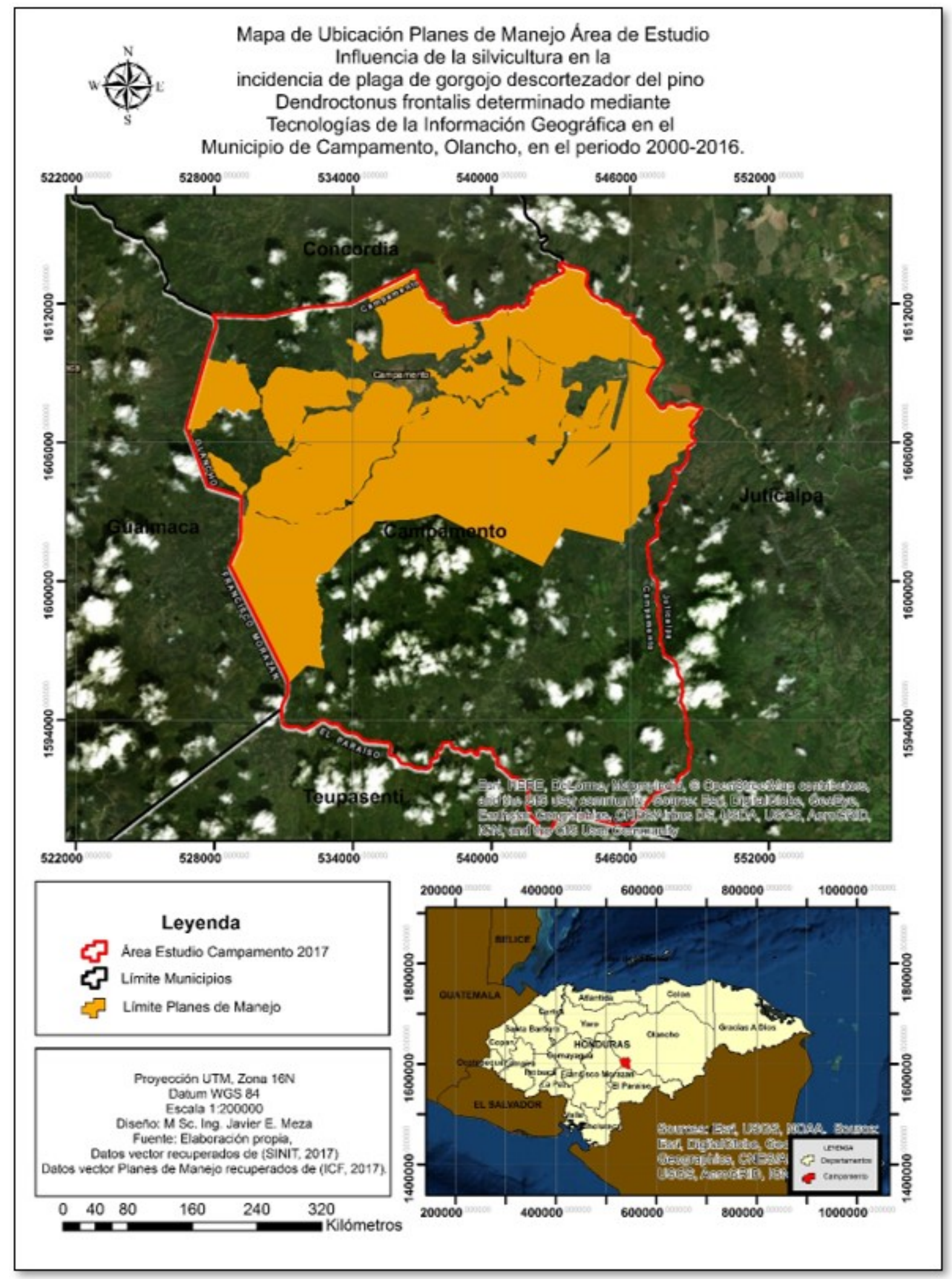

Figura 2: Mapa de ubicación planes de manejo área de estudio. 
Etapa III: análisis espacial utilizando herramientas SIG en el área de estudio.

Utilizando herramientas geoespaciales con software especializado en Sistemas de Información Geográfica se seleccionó el área de estudio de la capa vector del Sistema de Información Territorial SINIT. Así mismo, se realizaron varios cortes de las áreas de interés dentro del municipio de Campamento, para elaborar el mapa de uso actual y luego las consideraciones anteriores (ver figura 3). Posteriormente se realizó una selección de los planes de manejo dentro del área de interés.

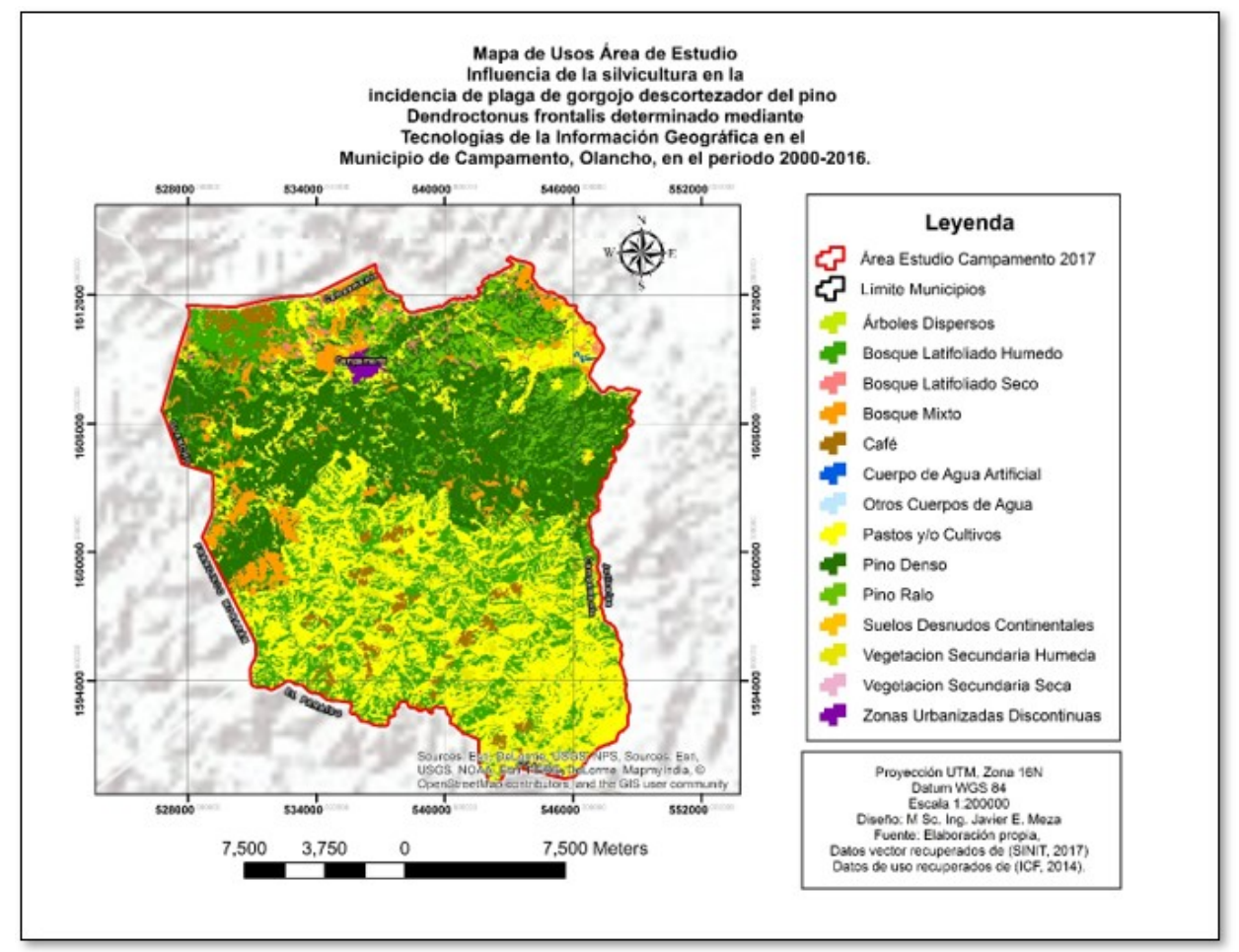

Figura 3: Mapa de usos de área de estudio.

A continuación, se realizó dos cortes, el primero de las áreas que se encuentran afectadas por la plaga del gorgojo descortezador dentro del área que se encuentra en el interior de los planes de manejo y el segundo corte del área afectada por la plaga del gorgojo descortezador que se encuentra fuera del área con régimen de manejo forestal. En ese mismo sentido se realizó la extracción o corte de la información de la capa del mapa de uso del 2014, proporcionada por el Instituto de Conservación Forestal. Dejando solamente el área de estudio que corresponde al municipio de Campamento.

Etapa IV: socialización y validación de resultados, esta permitió validar los resultados con los beneficiarios y actores de este estudio.

\section{Resultados}

Un aspecto importante que resaltar es que, del total del área de estudio, menos de la mitad (41.68\%) tiene cobertura de bosque de coníferas y bosque mixto que es sujeta a manejo forestal. Además, puede observarse que el $85.16 \%$ de esa área se encuentra bajo un régimen de manejo forestal, lo cual es muy positivo, tomando en consideración lo que muestra el análisis de los resultados (ver figura 4). 


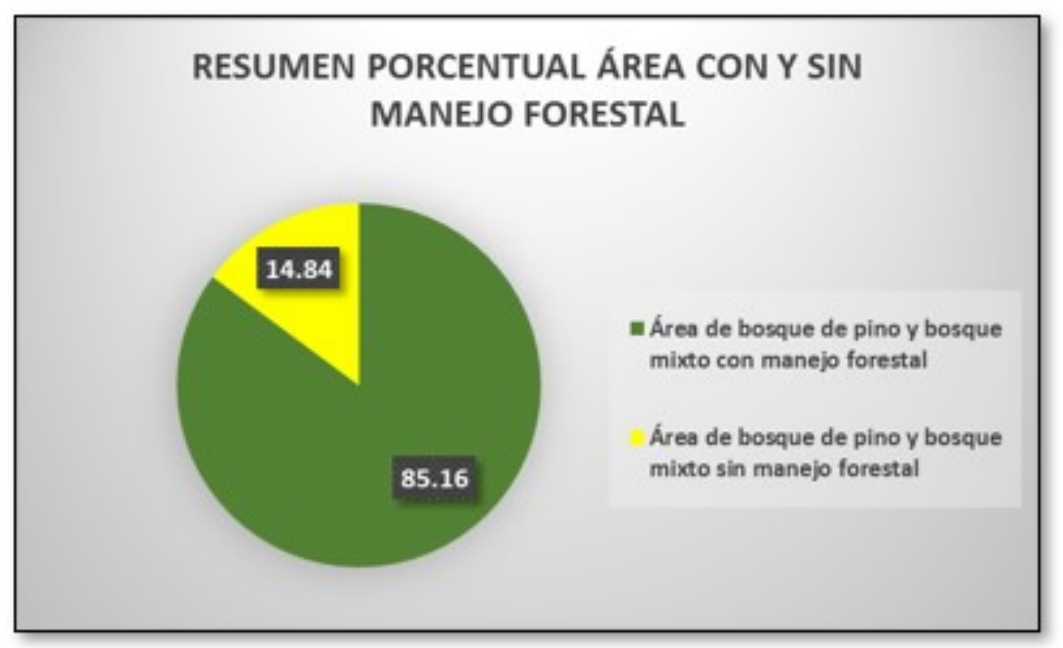

Figura 4: Gráfica resumen porcentual área con y sin manejo forestal.

Las figuras 4, 5, 6, 7 y 8 muestran los resultados de la investigación en donde se puede apreciar que hay una diferencia porcentual en el grado de afectación de plaga entre el bosque que fue manejado, bajo un régimen silvicultural y el bosque no fue manejado, los datos se pueden ver en la tabla 2, tabla 3 y tabla 4.

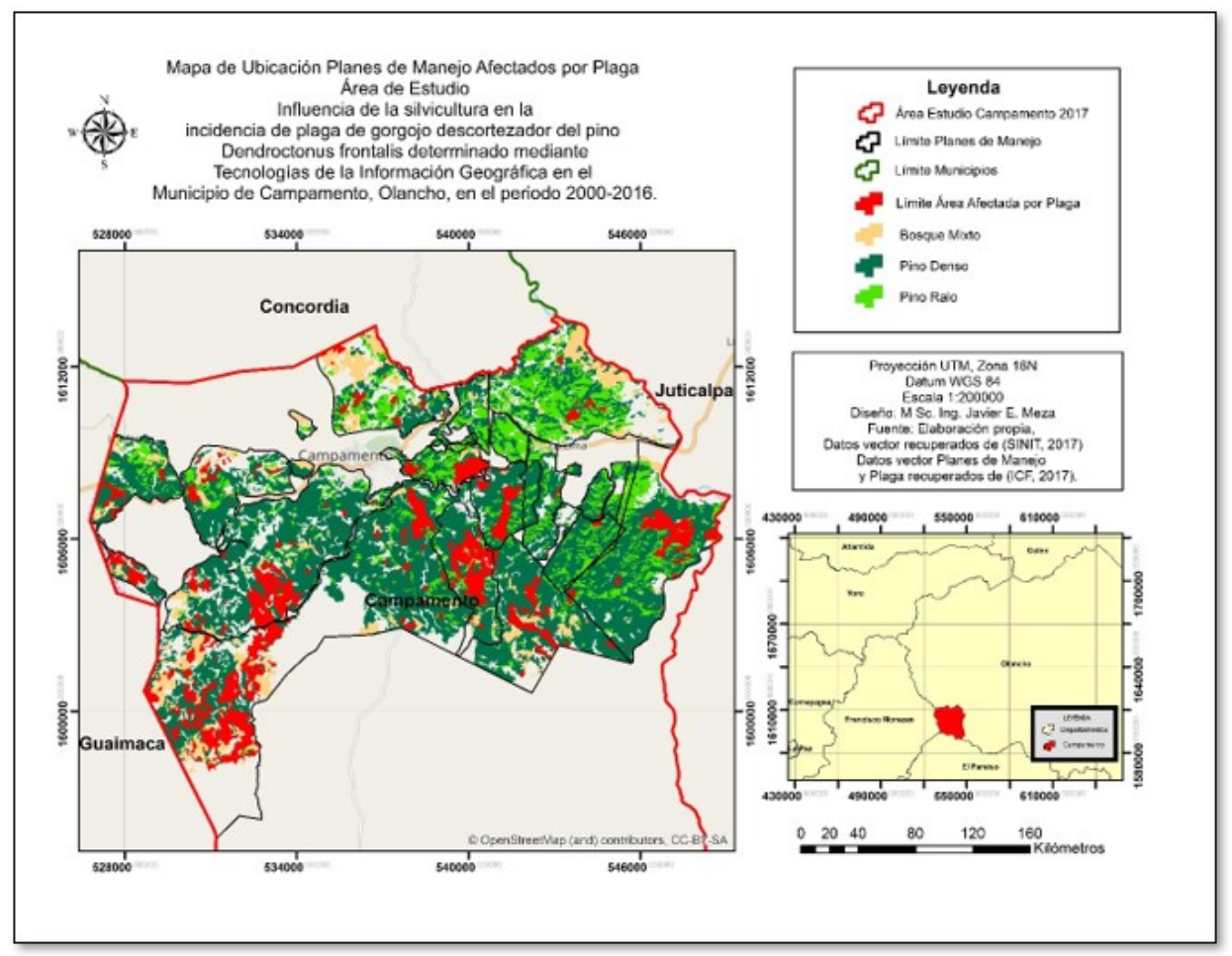

Figura 5: Mapa de ubicación planes de manejo afectados por el gorgojo descortezador. 


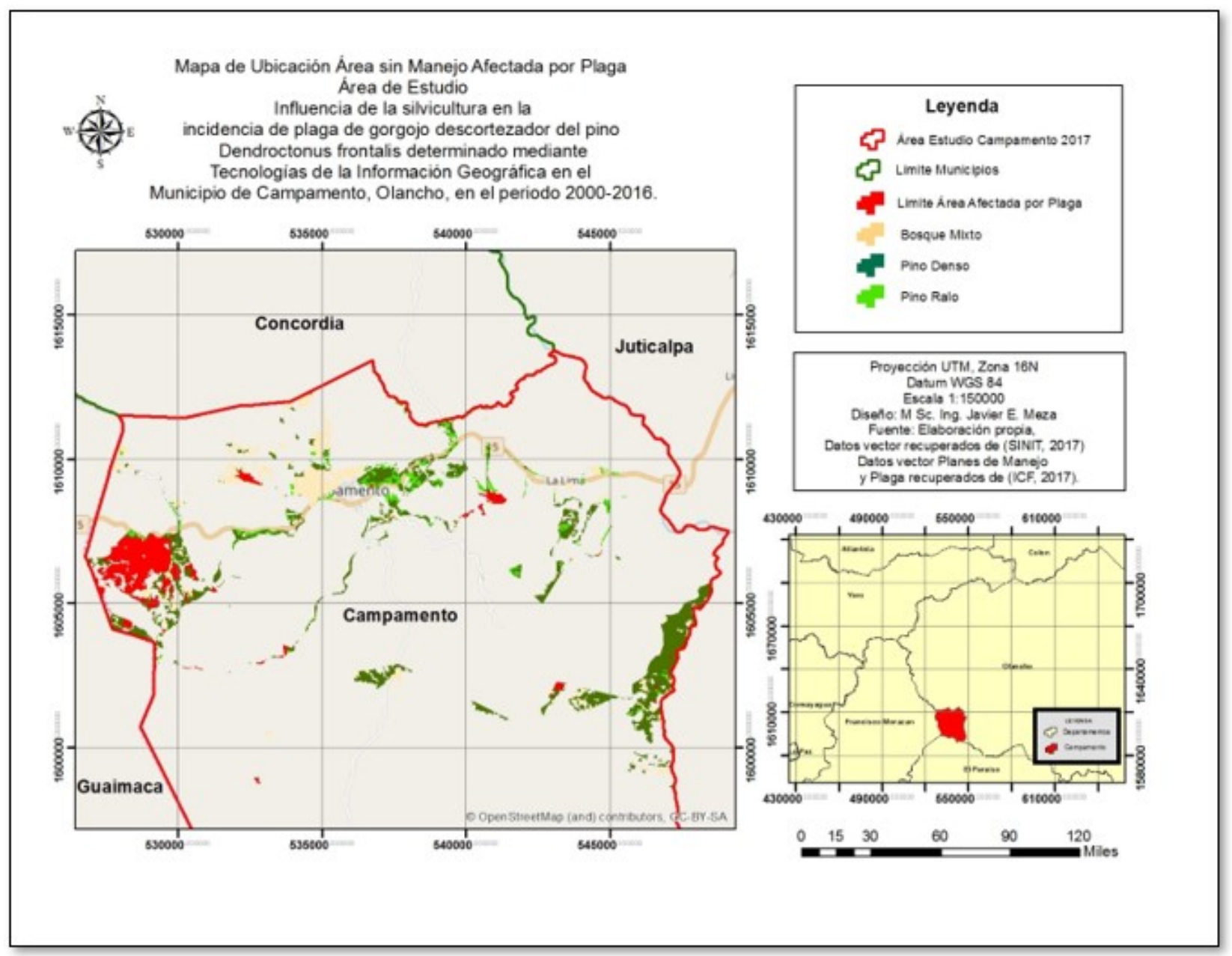

Figura 6: Mapa de ubicación área sin planes de manejo afectada por gorgojo descortezador.

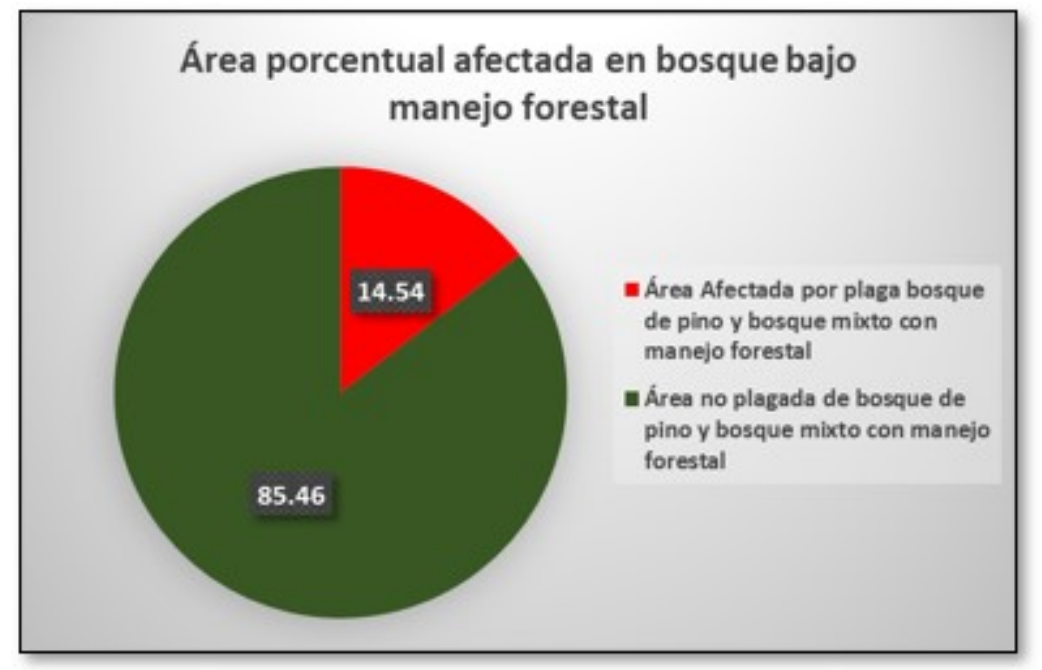

Figura 7: Gráfica área porcentual afectada en bosque con planes de manejo. 


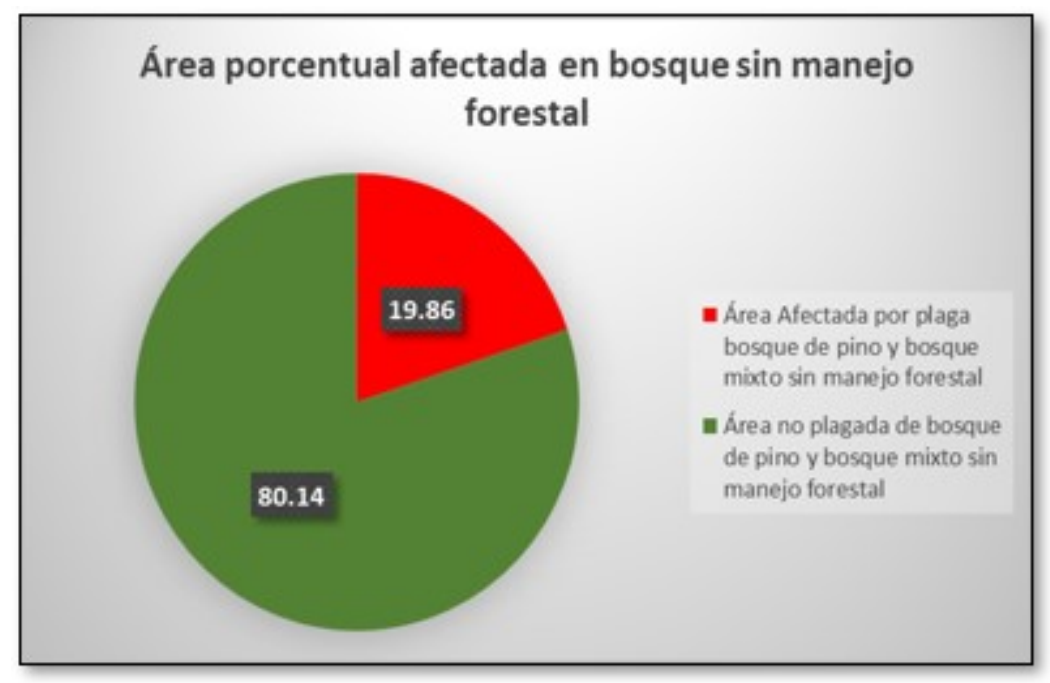

Figura 8: Gráfica área porcentual afectada en bosque sin planes de manejo forestal.

Tabla 2: Resumen área con y sin manejo forestal

\begin{tabular}{lcc}
\hline Descripción & Área (ha) & Porcentaje \\
\hline Área de bosque de pino y bosque mixto con manejo forestal & 13473.31 & 85.16 \\
Área de bosque de pino y bosque mixto sin manejo forestal & 2348.64 & 14.84 \\
\hline Total & $\mathbf{1 5 8 2 1 . 9 5}$ & $\mathbf{1 0 0 . 0 0}$ \\
\hline
\end{tabular}

Fuente:recuperado de Mapa Forestal ICF (2014)

Tabla 3: Resumen área afectada bajo manejo forestal

\begin{tabular}{lcc}
\hline Descripción & Área (ha) & Porcentaje \\
\hline Área Afectada por plaga bosque de pino y bosque mixto con manejo forestal & 1959.65 & 14.54 \\
Área no plagada de bosque de pino y bosque mixto con manejo forestal & 11513.66 & 85.46 \\
\hline Área total bosque de pino y bosque mixto con manejo forestal & $\mathbf{1 3 4 7 3 . 3 1}$ & $\mathbf{1 0 0 . 0 0}$ \\
\hline
\end{tabular}

Fuente: recuperado de datos vector ICF (2017)

Tabla 4: Resumen área afectada sin manejo forestal

\begin{tabular}{lcc}
\hline Descripción & Área (ha) & Porcentaje \\
\hline Área Afectada por plaga bosque de pino y bosque mixto sin manejo forestal & 466.52 & 19.86 \\
Área no plagada de bosque de pino y bosque mixto sin manejo forestal & 1882.12 & 80.14 \\
\hline Área de bosque de pino y bosque mixto sin manejo forestal & $\mathbf{2 3 4 8 . 6 4}$ & $\mathbf{1 0 0 . 0 0}$ \\
\hline
\end{tabular}

Fuente: recuperado de datos vector ICF (2017) 


\section{Discusión}

De acuerdo con los razonamientos que se han venido realizando se puede observar que el mayor grado de afectación se encuentra en el área de bosque de coníferas y en el bosque mixto que no ha recibido ningún tratamiento silvícola, lo que muestra en un primer análisis la importancia que tiene efectuar manejo forestal en estas áreas, para de alguna manera incidir en el impacto negativo que provoca el gorgojo descortezador de los pinos.

Hecha la observación anterior se hace necesario que la Administración Forestal del Estado fomente el manejo forestal a través de los incentivos al manejo forestal que se encuentran en la Ley Forestal Áreas Protegidas y Vida Silvestre y a la vez que se operativicen los mismos, para poder reducir el impacto negativo que provocan estos episodios de plaga.

De los anteriores planteamientos se deduce la necesidad de realizar este tipo de estudios, los cuales pueden desarrollarse a nivel local, regional y nacional para contar con nuevos elementos que vengan a ayudarnos a tomar decisiones e impulsar el desarrollo forestal del país, en un país en donde mas del $70 \%$ de la superficie es de vocación forestal, lo cual no se ve reflejado en el Producto Interno Bruto (PIB).

Los resultados muestran la necesidad de más datos y análisis, para los cual se requieren soluciones innovadoras y los sistemas de información geográfica representan una excelente opción ante esta problemática y sobre todo se necesita realizar más investigación y monitoreo constante para evitar los daños provocados por este fenómeno que provoca pérdidas económicas y ecologías al país.

\section{Conclusiones}

Existe una variación porcentual en la incidencia de la silvicultura de 19.86 \% en el área afectada por plaga bosque de pino y bosque mixto sin manejo forestal en el área de estudio equivalente a 466.52 hectáreas. Lo que muestra una variación porcentual que refleja una mayor afectación en áreas de bosque que no tienen un régimen silvicultural.

Hay una diferencia porcentual en la incidencia de la silvicultura de $14.54 \%$ en el área afectada por plaga bosque de pino y bosque mixto con manejo forestal en el área de estudio equivalente a 1959.65 hectáreas.

Existe una variación estadística o diferencia porcentual de $5.32 \%$ entre el bosque manejado y el bosque no manejado, lo que podría ser un indicador sobre la importancia de realizar silvicultura. No obstante, es importante realizar más estudios en otras áreas, para tener más datos al respecto.

Se puede observar que la densidad de los bosques de pino es una característica determinante en la incidencia de plaga. Es evidente entonces que resultara útil realizar otras investigaciones, para determinar la incidencia del factor densidad en la afectación del gorgojo descortezador del pino.

En los bosques que tienen planes de manejo y que hay tenido un régimen silvicultural intenso se puede observar una intensidad menor en el grado de afectación de la plaga del gorgojo descortezador del pino. Lo que es un indicador de la necesidad que existe de manejar los bosques, bajos regímenes silviculturales adecuados.

Área total bosque de pino y bosque mixto es de 15,821.95 que representa un $41.68 \%$ del área de estudio. De esta el $85.16 \%(13,473.31 \mathrm{ha})$ se encuentran bajo un régimen de manejo forestal y 2,438.64 ha que representan el $14.84 \%$ que se encuentran sin ningún manejo forestal, en esta última se encuentra el mayor porcentaje de afectación $19.86 \%$. 


\section{Referencias}

Alonso, D. (2015). Cómo calcular el área de un polígono con ArcGIS, QGIS y gvSIG. https:/ / mappinggis.com/2015/07/como-calcular-el-area-de-un-poligono-con-arcgis-qgis-y-gvsig/.

BID (2016). Proyecto Manejo Sostenible de Bosques. Proyecto BID HO-L1179.

CONADEH (2016). Informe Especial El gorgojo descortezador del pino y otras graves amenazas ambientales a la vida digna de los hondureños y hondureñas. Technical report, CONADEH.

FAO (2012). Guía para la Aplicación de Normas Fitosanitarias en el Sector Forestal.

Hernandez, A. (2016). Reporte Simulación con la Herramienta para la Evaluación de Suelos y Aguas Swat.

ICF (2014). Geoportal del Sector Forestal de Honduras, Instituto de Conservación Forestal. http://geoportal.icf.gob.hn/geoportal/main.

ICF (2015). Geoportal del Sector Forestal de Honduras, Instituto de Conservación Forestal. http://geoportal.icf.gob.hn/geoportal/main.

ICF (2016). Informe plagas.

ICF (2017). Unidad de Control de Plagas.

SINIT (2017). Sistema Nacional de Información Territorial. 